\title{
Mucuna pruriens Reduces Stress and Improves the Quality of Semen in Infertile Men
}

\author{
Kamla Kant Shukla1, Abbas Ali Mahdi ${ }^{1}$, Mohammad Kaleem Ahmad ${ }^{1}$, \\ Shyam Pyari Jaiswar ${ }^{2}$, Satya Narain Shankwar ${ }^{3}$ and Sarvada Chandra Tiwari ${ }^{4}$ \\ ${ }^{1}$ Department of Biochemistry, ${ }^{2}$ Department of Obstetric \& Gynaecology, ${ }^{3}$ Department of Urology and \\ ${ }^{4}$ Department of Psychiatry, King George's Medical University, Lucknow 226003, India
}

\begin{abstract}
The present investigation was undertaken to assess the role of Mucuna pruriens in infertile men who were under psychological stress. Study included 60 subjects who were undergoing infertility screening and were found to be suffering from psychological stress, assessed on the basis of a questionnaire and elevated serum cortisol levels. Age-matched 60 healthy men having normal semen parameters and who had previously initiated at least one pregnancy were included as controls. Infertile subjects were administered with $M$. pruriens seed powder $\left(5 \mathrm{~g} \mathrm{day}^{-1}\right)$ orally. For carrying out morphological and biochemical analysis, semen samples were collected twice, first before starting treatment and second after 3 months of treatment. The results demonstrated decreased sperm count and motility in subjects who were under psychological stress. Moreover, serum cortisol and seminal plasma lipid peroxide levels were also found elevated along with decreased seminal plasma glutathione (GSH) and ascorbic acid contents and reduced superoxide dismutase (SOD) and catalase activity. Treatment with $M$. pruriens significantly ameliorated psychological stress and seminal plasma lipid peroxide levels along with improved sperm count and motility. Treatment also restored the levels of SOD, catalase, GSH and ascorbic acid in seminal plasma of infertile men. On the basis of results of the present study, it may be concluded that $M$. pruriens not only reactivates the anti-oxidant defense system of infertile men but it also helps in the management of stress and improves semen quality.
\end{abstract}

Keywords: antioxidants - lipid peroxides - male infertility - Mucuna pruriens - psychological stress

\section{Introduction}

Infertility is a major public health concern and it is said to be the manifestation of one or more pathological conditions of either male or female origin. As many as $15 \%$ of couples have difficulty in conceiving, and the male factor is implicated as the cause in up to $50 \%$ of such cases. Moreover, in $\sim 10-15 \%$ of infertile couples, no apparent cause can be found and these cases are categorized under

For reprints and all correspondence: Prof. Dr Abbas Ali Mahdi, Department of Biochemistry, King George's Medical University,

Lucknow 226003, India. Tel: +91-9839011192; Fax: +91-522-2257539;

E-mail: mahdiaa@rediffmail.com 'unexplained infertility' (1). The problem of infertility is closely related to stress, as a couple, failing to achieve the expected goal of reproduction, experiences the feelings of frustration and disappointment. These feelings only compound in couples experiencing infertility related problems requiring prolonged efforts to conceive. Previous studies have indicated that stress, especially psychological stress, has a negative impact on various parameters associated with semen quality, including sperm concentration, motility and morphology. Other disturbances, such as impotence, sham ejaculation, retrograde ejaculation and oligospermia, have also been reported to be associated with psychological factors underlying male infertility (2). 
Chronic exposure to psychological stress is also known to cause a variety of patho-physiological changes in neuroendrocrine system, resulting in altered steroidogenesis and spermatogenesis. Prolonged stress also leads to increased blood cortisol level. An excess of this hormone, in turn, markedly affects the spermatogenesis, resulting in retention of cytoplasmic droplets and over-production of immature spermatozoa, which are reported to be the major source of reactive oxygen species (ROS) (3). Moreover, there are also reports that elevated psychological stress itself is associated with increased oxidant production and long-term exposure to psychological stressors may enhance the generation of ROS (4). Under normal circumstances, the ROS scavenging potential of the male reproductive tract and seminal fluid is maintained by adequate levels of enzymatic and non-enzymatic antioxidants, vitamins and minerals $(5,6)$. On the other hand, when the production of ROS is high, it may lead to oxidative damage to spermatozoa. ROS is also reported to have a negative effect on sperm functioning. The sperm plasma membrane is very sensitive to ROS, since it contains high levels of unsaturated fatty acids. The latter provide fluidity, which is necessary for sperm motility and acrosome reaction. Increased ROS level, as found during prolonged psychological stress, may lead to an imbalance between oxidant/anti-oxidant ratio, leading to increased lipid peroxidation, resulting in sperm membrane damage and its subsequent dysfunctioning (7).

Till date, appropriate treatment of idiopathic male infertility has not been found. However, in the ancient Indian system of medicine, the Ayurveda and Unani, as also the Chinese system of traditional remedies, some plants e.g. Mucuna pruriens, Tinospora cordiofolia, Asparagus racemosus, Withania somnifera, Orchis latifolia, Ocimum basilium and Tribulus terrestris etc. were used for the improvement of endurance against stress, general resistance against infections, retardation of the aging process and improving male sexual disorders, like psychogenic impotence and unexplained infertility (8-10).

Mucuna pruriens Linn. (Fabaceae), commonly known as 'cowhage plant' or 'kapikacho' or 'kevach' in Hindi, is the most popular drug in the Ayurvedic and Unani system of medicine. Its different preparations (from the seeds) are used for the management of several free radical-mediated diseases, such as rheumatoid arthritis, diabetes, atherosclerosis, nervous disorders and male infertility (11). There are reports that seed powder of $M$. pruriens helps in some way against stress, it increases secretion of semen and it acts as a restorative and an invigorating tonic or aphrodisiac in diseases characterized by weakness or loss of sexual power (12). It is also used in the management of Parkinsonism, as it is a good source of L-3, 4 dihydroxyphenyl alanine (L-DOPA) (13).

Seeds of $M$. pruriens also possess antioxidant, hypoglycemic, lipid lowering and neuroprotective activities (14). Its seeds contain the alkaloids, mucunine, mucunadine, mucunadinine, prurienidine and nicotine, besides $\beta$-sitosterol, glutathione, lecithin, vernolic acid and gallic acid. They posses a number of other bioactive substances, including tryptamine, alkylamines, steroids, flavonoids, coumarins, cardenolides and metals like magnesium, copper, zinc, manganese and iron (15). The present study was planned to investigate the effect of $M$. pruriens on semen quality and the seminal plasma levels of lipid peroxides and antioxidants in infertile men, who were under psychological stress.

\section{Materials and Methods}

\section{Chemicals}

Nitroblue tetrazolium Cat N-5514 (NBT), thiobarbituric acid Cat T-5500 (TBA), phenazine methosulphate Cat N-9625 (PMS), nicotinamide adenine dinucleotide Cat N-6754 (NADH), 5.5'-dithio bis 2- nitrobenzoic acid Cat D-5420 (DTNB), 4,5 methyl thiazol-2-yl 2,5 diphenyl tetra zolium bromide Cat M-2128 (MTT), nicotinamide adenine dinucleotide phosphate Cat N-7785 (NADPH) tricholoroacetic acid Cat T-8657 (TCA) and reduced glutathione Cat G-4251 (GSH) were purchased from Sigma Chemical Co., St Louis, MO, USA. Radioimmunoassay kit for estimation of serum cortisol was obtained from Diagnostic Product Corporation, Los Angeles, CA, USA. This kit with trade name Code-A-Count Cortisol, contains cortisol antibody coated polypropylene tubes (TC 01), a solution of iodinated cortisol ${ }^{125} \mathrm{I}$ cortisol (TC 02) as well as cortisol calibrators (TC 03-8) containing standard cortisol. All other reagents used were of high quality and analytical grade.

\section{Plant Material}

The seeds of plant $M$. pruriens were purchased from an authorized dealer in Lucknow, India. They were identified and authenticated by Dr M.M.A.A Khan, Senior Lecturer, Department of Botany, Shia P.G. College, Lucknow, India (Herbarium No. M-113 dated October 17, 2005). The seeds were dried under shade and made to a fine powder using laboratory mill.

\section{Study Protocol}

The study protocol was approved by the Ethical Committee of the King George's Medical University, Lucknow, (vide communication No.126/R-Cell-04, dated: May 14, 2004). Before enrolment in the study, written informed consent from each subject was obtained in response to a fully written and verbal explanation of the nature of the study. The potential participants, each with infertility persisting longer than a year, were clinically examined before being included in the study. 
Complete medical history of subjects and their female partners was also recorded. Subjects having diabetes, hypertension, arthritis, malignancy, tuberculosis, human immunodeficiency virus positive, infections, endocrinal disorders and on drugs and conditions known to influence oxidative stress and serum cortisol level were excluded.

\section{Subjects}

A total of 120 men, aged $30-38$ years, belonging to the same socioeconomic and ethnic status (Indo Aryan) and body mass index ranging from 19 to $24 \mathrm{~kg} \mathrm{~m}^{-2}$, were selected from the couples attending the Infertility Clinic of the Department of Obstetrics \& Gynecology and the Out Patient Department of Urology, King George's Medical University, Lucknow. All subjects were instructed not to take any nutritional supplement or vitamins and not to change their dietary habits during the course of study. This study was undertaken from January 2006 to December 2006.

\section{Sample Collection}

Semen samples were collected from subjects with 3-4 days of sexual abstinence. Semen analysis was carried out according to the World Health Organization guidelines (16). Venous blood samples were also withdrawn and serum separated for assessment of cortisol levels.

The prospective study included four parallel groups of subjects: The control group comprised of 60 age-matched healthy men who had previously initiated at least one pregnancy and exhibited a normal semen profile $\left(>20 \times 10^{6} \quad\right.$ spermatozoa $\mathrm{ml}^{-1} ; \quad>40 \%$ motility and $>40 \%$ normal morphology). Moreover, control subjects were also not under stress, as evidenced by the study questionnaire and normal serum cortisol level.

The study group comprised of 60 subjects who were under psychological stress (as assessed by the study questionnaire and on the basis of elevated serum cortisol levels). Study subjects were further divided into three subgroups on the basis of semen profiles and in each subgroup a minimum of 20 patients were included. These groups were (i) normozoospermic infertile men, defined as control group (ii), oligozoospermic infertile men $\left(<20 \times 10^{6}\right.$, spermatozoa ml ${ }^{-1}$, motility $>40 \%$ and $>40 \%$ normal morphology) and (iii) asthenozoospermic infertile men $\left(>20 \times 10^{6}\right.$ spermatozoa ml ${ }^{-1},<40 \%$ motility and $>40 \%$ normal morphology).

\section{Stress Questionnaire}

Psychological stress was assessed as per the protocol known as State Anxiety Inventory (17). All participants were asked to complete the questionnaire of the State Anxiety Inventory, as validated by Oner and Le Comple (18). In the questionnaire, subjects were asked to describe how they feel 'right now' by responding to 20 questions with a 4-point response format from 'not at all' (score 1) to 'extremely' (score 4). Total scores ranged from 20 to 80 , with higher scores indicating greater anxiety. This measure has been shown to have high reliability and good contrast validity.

\section{Treatment}

The infertile men were prescribed $M$. pruriens seed powder $\left(5 \mathrm{~g} \mathrm{day}^{-1}\right)$, orally, in a single dose for 3 months with a cup of skimmed milk (19). Semen samples were collected twice, i.e. first before administrating the medicine and second, after 3 months of treatment. All the semen morphological profiles were assessed within an hour of sample collection and biochemical profiles were evaluated within 2 days. During the course of study the patients were also monitored, on monthly basis, for liver functions.

\section{Preparation of Seminal Plasma}

Semen samples were collected by masturbation after 3-4 days of abstinence into sterile plastic containers for analysis. The semen volume was recorded and an aliquot was taken to assess sperm motility after at least $30 \mathrm{~min}$ given for liquefaction. There after, the semen samples were centrifuged at $1200 \times g$ in cold $\left(4^{\circ} \mathrm{C}\right)$ for $20 \mathrm{~min}$ for the separation of seminal plasma. The supernatant (seminal plasma) was again centrifuged at $10000 \times g$ in cold $\left(4^{\circ} \mathrm{C}\right)$ for $30 \mathrm{~min}$ to eliminate all possible contaminating cells and stored at $-20^{\circ} \mathrm{C}$ until analyzed. All biochemical estimations were carried out in seminal plasma.

\section{Biochemical Assay}

\section{Estimation of Lipid Peroxide Levels}

Seminal plasma $(0.2 \mathrm{ml})$ was mixed with $0.5 \mathrm{ml}$ glacial acetic acid. Subsequently, $0.5 \mathrm{ml}$ of $8 \%$ sodium dodecyl sulfate was added to the above reaction mixture. After mixing well $1.5 \mathrm{ml}$ of $0.8 \%$ TBA solution was added. The reaction mixture was kept in boiling water bath for $1 \mathrm{~h}$. After cooling to room temperature, $3.0 \mathrm{ml}$ of $n$-butanol was mixed, the reaction mixture was then centrifuged at $10000 \times g$ for $15 \mathrm{~min}$. The absorbance of clear butanol fraction obtained after centrifugation was read at $532 \mathrm{~nm}$ by UV-VIS double beam spectrophotometer (model No. 2203, serial No. 053, Systronics Instrumental Co. Hyderabad, India, year of purchase 2006). An appropriate standard made up of $2.5 \mathrm{nmol}$ malondialdehyde was run simultaneously (20).

\section{Assay of Catalase Activity}

Diluted hydrogen peroxide $(0.2 \mathrm{ml}$ of 20 -fold dilution, $30 \%$ $\mathrm{w} / \mathrm{v}$ ) and $2.5 \mathrm{ml}$ of $50 \mathrm{mM}$ phosphate buffer ( $\mathrm{pH} 8.2$ ) was 
added in a cuvatte. To the resultant, $0.02 \mathrm{ml}$ of seminal plasma, as enzyme source was added and mixed thoroughly. The decrease in the absorbance of reaction mixture due to catabolism of hydrogen peroxide was recorded at $240 \mathrm{~nm}$ after every $30 \mathrm{~s}$ by spectrophotometer (21).

\section{Assay of Superoxide Dismutase Activity}

Two reaction setups were run in parallel for superoxide dismutase (SOD) estimation. The tubes in the first setup (experimental) received $0.2 \mathrm{ml}(320 \mu \mathrm{M})$ NBT, $0.2 \mathrm{ml}$ $(10 \mu \mathrm{M})$ phenazine methosulfate, $2.0 \mathrm{ml}(0.16 \mathrm{mM})$ pyrophosphate buffer $\mathrm{pH}$ 9.2, 0.02 of seminal plasma as enzyme source. The tubes in the second setup (reference) received the entire above reagents except the enzyme source. The reaction was started simultaneously in both sets by the addition of $0.2 \mathrm{ml}(160 \mu \mathrm{M})$ of NADH. After an interval of $90 \mathrm{~s}, 1 \mathrm{ml}$ of glacial acidic acid was added to each reaction tube. The reference tubes were then added with the same amount of enzyme source, and absorbance was read at $560 \mathrm{~nm}$ against a blank on spectrophotometer (22).

\section{Estimation of Reduced Glutathione}

Seminal plasma $0.2 \mathrm{ml}$ was mixed with $3.0 \mathrm{ml}$ of $5 \%(\mathrm{w} / \mathrm{v})$ TCA reagent and allowed to stand for $5 \mathrm{~min}$, proteins were precipitated and filtered out. Later, $2.0 \mathrm{ml}$ of filtrate was added to $4.0 \mathrm{ml}$ of $0.3 \mathrm{M}$ phosphate buffer $\mathrm{pH} 7.4$ and $1 \mathrm{ml}$ of DTNB (1\% w/v aqueous sodium citrate). A blank was prepared in a similar manner using distilled water in place of the filtrate. An appropriate standard solution of $0.1 \mathrm{ml} \mathrm{GSH}(10 \mu \mathrm{mol})$ was also run simultaneous. The pale yellow color developed was read immediately at $412 \mathrm{~nm}$ by spectrophotometer (23).

\section{Estimation of Ascorbic Acid}

Phosphate citrate buffer $1.5 \mathrm{ml}$ (pH 8.2), $0.2 \mathrm{ml}$ PMS, $0.2 \mathrm{ml}$ MTT and $0.1 \mathrm{ml}$ of seminal plasma were added in a test tube and incubated at $37^{\circ} \mathrm{C}$ for $15 \mathrm{~min}$. The reaction was stopped by adding $0.5 \mathrm{ml}$ acetic acid. An appropriate standard solution of ascorbic acid $(0.1 \mathrm{ml})$ was also run simultaneously. Brown color was developed and measured by spectrophotometer at $578 \mathrm{~nm}$ (24).

\section{Estimation of Serum Cortisol Levels}

Serum cortisol levels were assessed by radioimmunoassay method (PC-RIA-MAS, Gamma Counter, StratecGermany, serial No. 2486000031 year of purchase2002) (25).

\section{Statistical Analysis}

Normal healthy fertile men and infertile groups were compared using one-way ANOVA analysis of variance followed by Dunnett test. Infertile groups, before and after treatment, were compared with paired $t$-test. A probability $P$-value of $<0.05(P<0.05)$ was considered statistically significant. The statistical analysis was performed on commercial software INSTAT 3.0, a demo version (Graph Pad Software, San Diego, CA).

\section{Results}

\section{Effect of M. pruriens on Semen Parameters in Infertile Men}

General semen characteristics of different groups of subject, before and after treatment with $M$. pruriens, are depicted in Fig. 1. In normal healthy fertile men (nonstress, control group), the mean sperm concentration was $57.0 \pm 8.4 \times 10^{6} \mathrm{ml}^{-1}$, motility was $57.0 \pm 5.4 \%$ and liquefaction time was $21.5 \pm 2.5 \mathrm{~min}$. The sperm concentration and motility in all under stress infertile groups as compared with controls were found decreased. The maximum decrease in sperm concentration was observed in oligozoospermic group $(-87 \% ; P<0.001)$ and motility of spermatozoa in asthenozoospermic patients $(-78 \%$; $P<0.001)$. Treatment of these infertile males with $M$. pruriens seed powder ( $5 \mathrm{~g} /$ day $)$ for 3 months showed significant reversal of the above findings. Our results show that sperm concentration was most significantly increased in oligozoospermic patients $(+688 \% ; P<0.001)$ and sperm motility was significantly increased in asthenozoospermic patients $(+32 \% ; P<0.05)$.

\section{Effect of M. pruriens on Stress Parameters in Infertile Men}

Stress scores, elaborated on the basis of the questionnaire, were found significantly high in infertile subjects (Table 1). Similarly, the morning serum cortisol levels were found elevated in normozoospermic $(+38 \% ; P<0.01)$, oligozoospermic $(+110 \% ; P<0.01)$ and asthenozoospermic $(+171 \% ; P<0.01)$ infertile men as compared with control subjects $\left(10.2 \pm 0.2 \mu \mathrm{g} \mathrm{dl}^{-1}\right)$. After treatment with $M$. pruriens, serum cortisol levels were decreased in normozoospermic $(-25 \% ; P<0.001)$, oligozoospermic $(-81 \% ; \quad P<0.001)$ and asthenozoospermic $(-55 \%$; $P<0.001)$ patients. Moreover, the mean serum cortisol levels observed at $1600 \mathrm{~h}$, in control group were $5.5 \pm 0.6 \mathrm{~g} \mathrm{dl}^{-1}$. But these levels in normozoospermic (99\%; $P<0.01)$, oligozoospermic $(162 \% ; P<0.01)$ and asthenozoospermic $(231 \% ; P<0.01)$ were found to be increased. After treatment with $M$. pruriens, significant reversal of serum cortisol levels in normozoospermic $(40 \% ; P<0.001)$, oligozoospermic $(46 \% ; P<0.001)$ and asthenozoospermic $(49 \% ; \quad P<0.001)$ was observed. The intra and inter assay variances of serum cortisol were $<6 \%$ and $<10 \%$, respectively, which was found to be highly significant. 


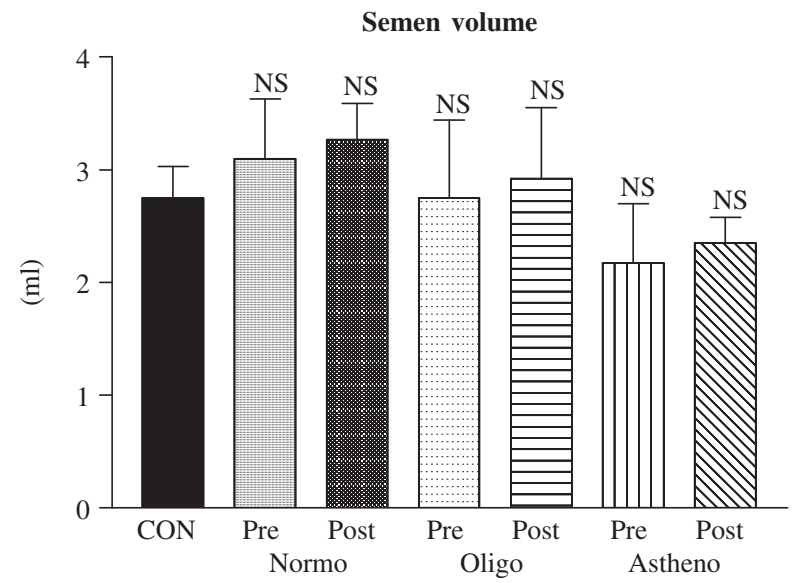

Subjects

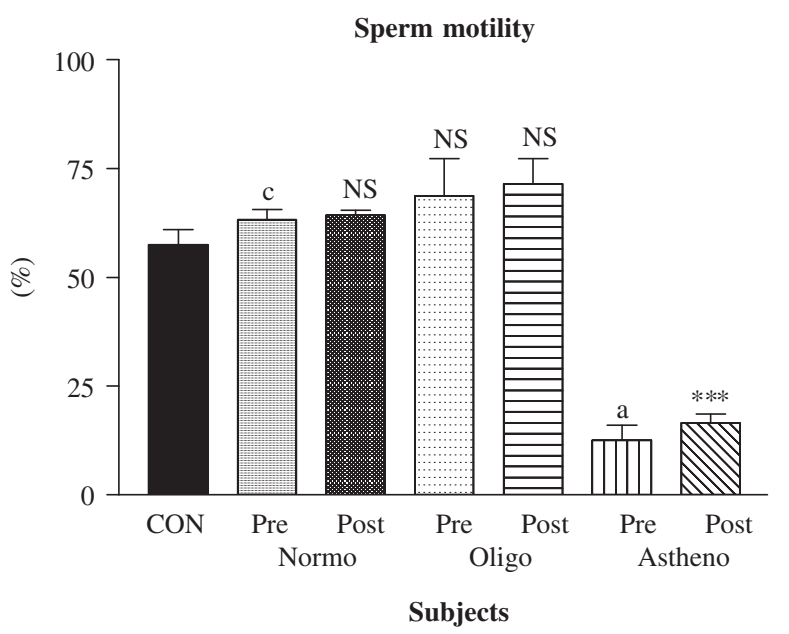

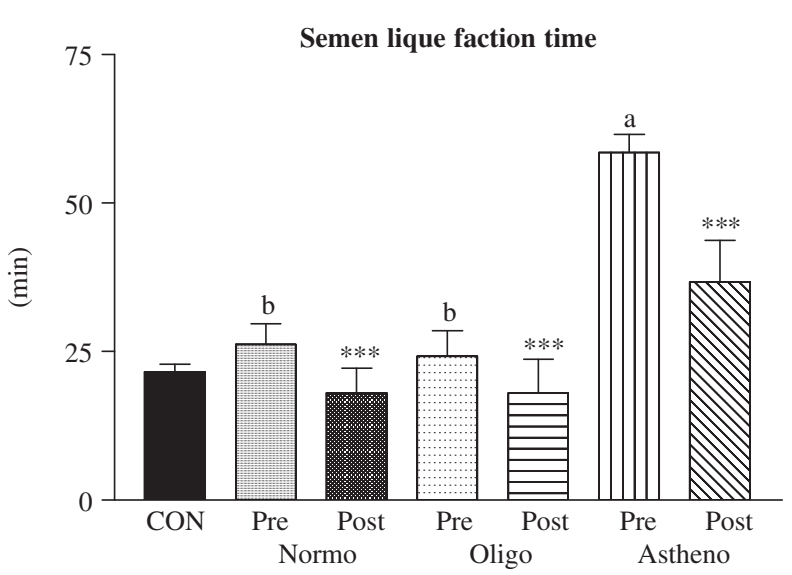

Subjects

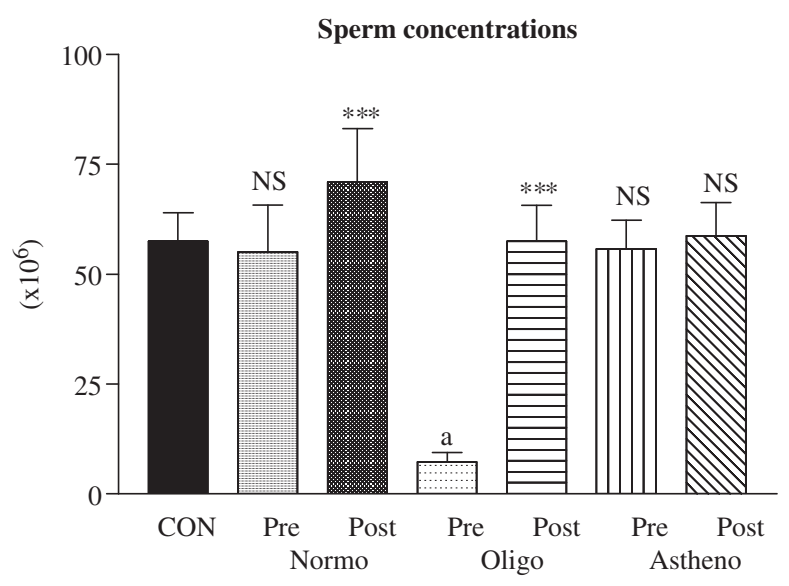

Subjects

Figure 1. Semen profiles of 'under stress' infertile men treated with $M$. pruriens. CON = Control; Pre = Pre-treatment; Post $=$ Post-treatment; Normo $=$ Normozoospermic; $\quad$ Oligo $=$ Oligozoospermic; $\quad$ Astheno $=$ Asthenozoospermic. $\quad$ Each bar represents mean \pm SD. Significance: $\mathrm{a}=\mathrm{P}<0.001, \mathrm{~b}=\mathrm{P}<0.01, \mathrm{c}=\mathrm{P}<0.05$; compared with controls, $* * *=\mathrm{P}<0.001$; compared with pre treatment subjects, NS $=$ Not significant.

Table 1. State anxiety score and serum cortisol levels in infertile men before and after treatment with M. pruriens

\begin{tabular}{lcccc}
\hline Stress parameter & Non stress (Control) & Psychological stress & & \\
\cline { 3 - 5 } & & Normozoospermic & Oligozoospermic & Asthenozoospermic \\
\hline State anxiety score & $41.0 \pm 8.0$ & $50.1 \pm 11.8^{\mathrm{a}}$ & $58.6 \pm 5.6^{\mathrm{a}}$ & $62.2 \pm 10.4^{\mathrm{a}}$ \\
Serum cortisol $(\mu \mathrm{g} / \mathrm{dl})(0800 \mathrm{~h})$ & $10.2 \pm 0.2$ & Pre $14.1 \pm 3.0^{\mathrm{b}}$ & Pre $21.5 \pm 0.7^{\mathrm{b}}$ & ${\text { Pre } 28.0 \pm 1.0^{\mathrm{b}}}$ \\
& & Post $11.6 \pm 0.4^{* * *}$ & Post $10.4 \pm 0.5^{* * *}$ & Post $12.6 \pm 2.0^{* * *}$ \\
Serum cortisol $(\mu \mathrm{g} / \mathrm{dl})(1600 \mathrm{~h})$ & $5.0 \pm 0.6$ & Pre $10.1 \pm 1.6^{\mathrm{b}}$ & Pre $13.3 \pm 4.6^{\mathrm{b}}$ & Pre $16.8 \pm 1.3^{\mathrm{b}}$ \\
& & Post $6.0 \pm 0.3^{* * *}$ & Post $7.3 \pm 2.1^{* * *}$ & Post $8.5 \pm 0.8^{* * *}$ \\
\hline
\end{tabular}

Values are expressed as mean $\pm \mathrm{SD}$.

Significance: State anxiety score: ${ }^{\text {a } P}<0.01$, as compared with the control group.

Cortisol: ${ }^{b} P<0.01$, as compared with the control group; ${ }^{* * *} P<0.001$, as compared with the pre treatment group.

Pre $=$ Pre-treatment; Post $=$ Post-treatment

\section{Effect of M. pruriens on Lipid Peroxide Levels and Antioxidant Parameters in Infertile Men}

The lipid peroxide level in seminal plasma of control healthy men was $2.2 \pm 0.3 \mathrm{nmol} \mathrm{MDA} \mathrm{ml}{ }^{-1}$. On the other hand, it was found increased in 'under stress' normozoospermic $(+55 \% ; \quad P<0.01), \quad$ oligozoospermic $(+48 \%$; $P<0.01)$ and asthenozoospermic $(+49 \% ; \quad P<0.01)$ subjects (Table 2). After treatment with M. pruriens, the levels of lipid peroxides were reversed significantly in normozoospermic $(-34 \%: P<0.001)$, oligozoospermic $(-27 \% ; P<0.001)$ and asthenozoospermic men $(-28 \%$; $P<0.001)$. We also observed that SOD activity in seminal plasma of control group was $8.1 \pm 0.5$ unitmg $^{-1}$ protein. However, this enzyme was found significantly suppressed 
Table 2. Biochemical parameters of under stress infertile men before and after treatment with $M$. pruriens

\begin{tabular}{|c|c|c|c|c|c|c|c|}
\hline \multirow[t]{2}{*}{ Biochemical Parameter } & \multirow{2}{*}{$\begin{array}{l}\text { Non-stress } \\
\text { (Control) }\end{array}$} & \multicolumn{6}{|c|}{ Psychological stress } \\
\hline & & Pre-treatment & Post-treatment & Pre-treatment & Post-treatment & Pre-treatment & Post-treatment \\
\hline $\begin{array}{l}\text { Superoxide Dismutase } \\
\text { (Unit } \mathrm{mg}^{-1} \text { protein) }\end{array}$ & $8.1 \pm 0.7$ & $6.5 \pm 0.5^{\mathrm{b}}$ & $6.7 \pm 0.5^{\mathrm{NS}}$ & $5.4 \pm 0.4^{\mathrm{b}}$ & $7.2 \pm 0.7^{* * *}$ & $5.7 \pm 0.5^{\mathrm{b}}$ & $6.8 \pm 0.7 * * *$ \\
\hline Catalase (Unitmg ${ }^{-1}$ protein) & $9.2 \pm 0.8$ & $8.6 \pm 0.7^{\mathrm{c}}$ & $9.3 \pm 0.8 * *$ & $8.8 \pm 0.6^{\mathrm{c}}$ & $9.2 \pm 0.3^{*}$ & $6.5 \pm 0.9^{\mathrm{c}}$ & $7.2 \pm 0.7^{* * *}$ \\
\hline Glutathione $\left(\mathrm{mg} \mathrm{dl}^{-1}\right)$ & $1.6 \pm 0.5$ & $1.3 \pm 0.2^{\mathrm{c}}$ & $1.6 \pm 0.3 * *$ & $1.3 \pm 0.2^{\mathrm{c}}$ & $1.6 \pm 0.5^{* *}$ & $1.3 \pm 0.3^{\mathrm{c}}$ & $1.6 \pm 0.3 * *$ \\
\hline Fructose $\left(\mathrm{mg} \mathrm{ml}^{-1}\right)$ & $2.6 \pm 0.4$ & $2.2 \pm 0.2^{\mathrm{b}}$ & $2.6 \pm 0.4^{\mathrm{NS}}$ & $1.6 \pm 0.4^{\mathrm{b}}$ & $2.4 \pm 0.3^{* *}$ & $1.1 \pm 0.3^{\mathrm{b}}$ & $1.3 \pm 0.3^{* *}$ \\
\hline
\end{tabular}

Values are expressed as mean $\pm \mathrm{SD}$.

Significance: ${ }^{\mathrm{a}} P<0.001,{ }^{\mathrm{b}} P<0.01,{ }^{\mathrm{c}} P<0.05$ as compared with the control group.

$* * * P<0.001,{ }^{* *} P<0.01, * P<0.05$ as compared with the pre treatment group, ${ }^{N S}$ Not significant.

in different groups of infertile men, who were under stress; such as normozoospermic $(-19 \% ; P<0.001)$, oligozoospermic $(-33 \% ; \quad P<0.001)$ and asthenozoospermic $(-29 \% ; P<0.001)$. Treatment with $M$. pruriens increased the activity of SOD in normozoospermic $(+3 \% ; P<0.01)$, oligozoospermic $(+33 \% ; P<0.01)$ and asthenozoospermic $(+18 \% ; P<0.01)$ men. Similarly, catalase activity in seminal plasma of 'under stress' asthenozoospermic men was found significantly reduced $(-30 \% ; P<0.05)$, as compared with healthy fertile men. Treatment with $M$. pruriens enhanced the activity of the aforementioned enzyme in all the infertile men.

Ascorbic acid levels in seminal plasma of control group were $2.3 \pm 0.2 \mathrm{mg} \mathrm{dl}^{-1}$. On the other hand, these levels were found decreased in different groups of infertile men who were under stress, i.e-normozoospermic $(-9 \% ; P<0.01)$, oligozoospermic $(-23 \% ; P<0.01)$ and asthenozoospermic $(-36 \% ; P<0.01)$. After treatment with $M$. pruriens the levels of ascorbic acid were found increased in normozoospermic $(+11 \% ; \quad P<0.5), \quad$ oligozoospermic $(+33 \% ; \quad P<0.01)$ and asthenozoospermic $(+36 \%$; $P<0.01)$ men (Table 2). Similarly, GSH content in seminal plasma of under stress normozoospermic $(-13 \%$; $P>0.05)$, oligozoospermic $(-20 \% ; P>0.05)$ and asthenozoospermic $(-47 \% ; P<0.05)$ infertile men was found decreased as compared with control group. Treatment with the drug restored the levels of GSH in normozoospermic $(+19 \% ; P<0.05)$, oligozoospermic $(+18 \% ; P<0.05)$ and asthenozoospermic $(+22 \% ; P<0.05)$ men.

\section{Discussion}

We observed that oral administration of $M$. pruriens to infertile men for 3 months not only resulted in general improvement in sperm count and motility but it also led to significant reduction in the level of psychological stress, as assessed by a questionnaire and serum cortisol levels. Male fertility and reproduction are known to be affected by various kinds of stressful conditions, including psychological stress (26). The autonomic nervous system and adrenal hormones participate in stress response, which also affects steroidogenesis and spermatogenesis (27). We report elevated serum cortisol levels in infertile men, who were under psychological stress. The latter causes stimulation of hypothalamic-pituitary-adrenal axis (HPA) leading to the release of the corticotropin releasing hormone (CRF), adrenocorticotropin hormone (ACTH) and cortisol. Chronically increased cortisol level, as seen during prolonged stress, may reduce the functional activity of leuteinizing hormone-release hormone (LHRH) pulse generator, which may lead to decrease in gonadotropin and testosterone levels $(26,28)$. Moreover, long-term psychological stress may also decrease the concentration of catchecholamines, like dopamine, noradrenalin, 5,6 , dihydroxy phenyl acetic acid (DOPAC) and homovanillic acid (HVA) in brain. Decrease in the activity of dopaminergic neurons is also known to affect the fertility, sperm count and motility. There are reports that $M$. pruriens is also rich in L-DOPA, besides having several other alkaloids and flavonoids. Therefore, our results demonstrating reduction in psychological stress following administration of $M$. pruriens may be linked to high L-DOPA content of this herb (29).

Our results also demonstrate that lipid peroxide levels were significantly high in the seminal plasma of subjects who were under stress, which may be due to increased oxidative stress. Psychological stress is known to be associated with increased oxidant production and longterm exposure to stress may lead to peroxidation of polyunsaturated fatty acids of sperm membrane, resulting in unfavorable alterations in sperm structure and function $(6,7)$. Moreover, we also observed that in infertile men who were under psychological stress, there were low seminal plasma SOD and catalase activities and reduced levels of glutathione and ascorbic acid. But there was improvement in these enzymes and molecule levels following treatment with $M$. pruriens. The improvement in anti-oxidant content following treatment may be due 
to the reduction of oxidative stress. As stated earlier, $M$. pruriens is reported to contain many bioactive constituents, including alkaloids, coumarins, flavonoids and alkylamines etc. which play an important role in increasing the antioxidant capacity of treated men (15). Furthermore, reduced stress and reactivation of antioxidants might have in turn lead to reduction in seminal plasma lipid peroxide content. Our results are also in concurrence with earlier reports that $M$. pruriens is a known adaptogen and its alcoholic extract reduces lipid peroxidation and maintains the levels of glutathione and SOD activity $(30,31)$.

$M$. pruriens seeds are rich source of L-DOPA and its metabolites, which include epinephrine and norepinephrine. Therefore, an increase in dopamine level in the brain following $M$. pruriens treatment may not only induce the activation of sexual behavior but it may also increase plasma testosterone level. It has been reported recently that L-DOPA and its metabolite dopamine stimulate the hypothalamus and forebrain to secrete gonadotropinreleasing hormone $(\mathrm{GnRH})$ (32). This, in turn, upregulates the anterior pituitary gland to secrete follicle stimulating hormone (FSH) and luteinizing hormone (LH) causing increased synthesis of testosterone by Leydig cells of the testis $(33,34)$. Furthermore, spermatogenesis is controlled by the hypothalamus and anterior pituitary working together. On the basis of the aforestated facts, it may be proposed that increased dopamine level in the brain may not only optimize the release of hormones, including testosterone, leading to increased sexual drive and improved performance, but it may also accomplish reduction of psychological stress. Moreover, treatment with $M$. pruriens may also contribute to proper functioning of male genital system and facilitate sperm transport, contraction of seminal vesicles and inhibition of lipid peroxidation of spermatozoa (35).

On the basis of results of the present study and as gleaned by earlier reports, it may be concluded that $M$. pruriens not only helps in reducing psychological stress, but also improves semen quality as it restores antioxidant levels and reduces lipid peroxide content.

\section{Acknowledgements}

Authors acknowledge with thanks the financial support from Indian Council of Medical Research, New Delhi in the form of Ad-hoc research scheme No. 5/10/8/2004RHN. Authors acknowledge with thanks the help and assistance of Prof. Mahdi Hasan (Department of Anatomy), Prof. R.K. Singh (HOD, Biochemistry) and Dr Ramesh Chander (Department of Biochemistry). Moreover, thanks are also due to Dr Farzana Mahdi, Director Academics, Era's Lucknow Medical College and Hospital, Lucknow, for providing facilities for some of the laboratory work. We would also like to thank $\mathrm{Mr}$ M.P.S. Negi, Biometry and Statistics Division,
CDRI, Lucknow for providing assistance in statistical analysis of data and preparation of graphs.

\section{References}

1. Sharlip ID, Jarow JP, Belker AM, Lipshultz LI, Sigman M, Thomas AJ, et al. Best practice policies for male infertility. Fertil Steril 2002;77:873-82.

2. McGrady AV. Effects of psychological stress on male reproduction: a review. Arch Androl 1984;13:1-7.

3. Gill GE, Ollero M, Lopez MC. Differential production of reactive oxygen species by subsets of human spermatozoa at different stages of maturation. Hum Reprod 2001;16:1922-30.

4. Wang L, Muxin G, Nishida H, Shirakawa C, Sato S, Konishi T. Psychological stress-induced oxidative stress as a model of subhealthy condition and the effect of TCM. Evid Based Complement Alternat Med 2006;4:195-202.

5. Eskiocak S, Gozen AS, Kilic AS, Molla S. Association between mental stress and some antioxidant enzymes of seminal plasma. Indian J Med Res 2005;122:491-6.

6. Mahdi AA, Bano F, Singh R, Singh RK, Siddiqui MS, Hasan M. Seminal plasma superoxide dismutase and catalase activities in infertile men. Med Sci Res 1999;27:201-3.

7. Sikka SC. Relative impact of oxidative stress on male reproductive function. Curr Med Chem 2001;8:851-62.

8. Dahanukar SA, Hazra A. Ayurveda unravelled. In: Dahanukar SA (ed). Heal with Herbs. New Delhi, India: Publications and Information Directorate, Council of Scientific and Industrial Research; 1995, 53-74.

9. Kaphle K, Wu LS, Yang NYJ, Lin JH. Herbal medicine research in Taiwan. Evid Based Complement Alternat Med 2006;3:149-55.

10. Yang NYJ, Kaphle K, Wang PH, Jong DS, Wu LS, Lin JH. Effect of aqueous extracts of betel quid and its constituents on testosterone production by dispersed mouse interstitial cells. Am J Chin Med 2004;32:705-15.

11. Rajeshwar Y, Kumar GPS, Gupta M, Mazumder UK. Studies on in vitro antioxidant activities of methanol extract of Mucuna pruriens (Fabaceae) seeds. Eur Bull Drug Res 2005;13:31-9.

12. Kumar KVA, Srinivasan KK, Shanbhag T, Rao SG. Aphrodisiac activity of the seeds of Mucuna pruriens. Indian Drug 1994;31:321-7.

13. Molloy SA, Rowan EN, O'Brien JT, McKeith IG, Wesnes K, Burn DJ. Effect of levodopa on cognitive function in Parkinson's disease with and without dementia and dementia with Lewy bodies. J Neurol Neurosurg Psychiatr 2006;77:1323-8.

14. Sharma ML, Chandhoke N, Ray Ghatak BJ, Jamwal KS, Gupta OP, Singh GB. Pharmacological screening of Indian medicinal plants. Indian J Exp Biol 1978;16:228-35.

15. Misra L, Wagner H. Extraction of bioactive principle from Mucuna pruriens seeds. Indian J Biochem Biophys 2007;44:56-60.

16. World Health Organization. Laboratory manual for the examination of human semen and sperm cervical mucus interaction, 4th edn. New York: Cambridge University Press, 1999.

17. Spielberger CD, Gorsuch RL, Lushene RE. STAI manual for the State-Trait Anxiety Inventory. Palo Alto, CA, USA: Consulting Psychologist Press, 1970.

18. Oner N, Le Comple A. DurumLuluk-Surekilik Kaygi Envanteri: Ei Kitabi. Istanbul, Turkey: Bogazici Universitesi Matbaasi, 1998.

19. Singh D. Konch (Kiwach). In: Singh D (ed). Unani Dravvyagunadarsh, Vol. 2, Varanasi, India: Jivan Shiksha Mudralay Limited, Lucknow, Uttar Pradesh, India, 1974, 101-2.

20. Ohkawa H, Ohisha N, Yagi K. Assay of lipid peroxides in animal tissue by thiobarbituric acid reaction. Anal Biochem 1979;5:351-8.

21. Aebi H. Catalase. In: Bergmeyer HU (ed). Methods of Enzymatic Analysis, Vol. 2. New York: Academic Press Inc, 1974, 673-84.

22. McCord JM, Fridovich I. Superoxide dismutase: an enzyme function for erythrocuprin. J Biol Chem 1969;244:6049-55.

23. Hissin PJ, Hilf R. A fluorometric method for determination of oxidized and reduced glutathione in tissues. Anal Biochem 1976;74:214-26.

24. Gavella M, Lipovac V, Vucic M, Rocic B. Evaluation of ascorbate and urate antioxidant capacity in human semen. Andrologia 1997;29:29-35. 
25. Foster LB, Dunn RT. Single antibody technique for radioimmunoassay of cortisol in unextracted serum or plasma. Clin Chem 1974;20:365-8.

26. Nagro-Vilar A. Stress and other environmental factors affecting fertility in men and women; overview. Env Health Prospect Suppl 1993;101 (Suppl 2):59-64.

27. Hardy MP, Gao HB, Dong Q, Ge R, Wang Q, Chai WR, et al. Stress hormone and male reproductive function. Cell Tissue Res 2005;322:147-53.

28. Axelrod J, Reisine TD. Stress hormones: their interaction and regulation. Science 1984;224:452-6.

29. Sato Y, Suzuki N, Horita H, Wada H, Shibuya A, Adachi H, et al. Effect of long term psychological stress on sexual behavior and brain catecholamine levels. J Androl 1996;17:83-90.

30. Tripathi YB, Upadhyay AK. Antioxidant property of Mucuna pruriens. Curr Sci 2001;80:1377-8.
31. Tripathi YB, Upadhyay AK. Effect of the alcohol extract of the seeds of Mucuna pruriens on free radicals and oxidative stress in albino rats. Phytother Res 2002;16:534-8.

32. Vermes I, Toth EK, Telegdy G. Effects of drugs on brain neurotransmitter and pituitary testicular function in male rats. Horm Res 1979;10:222-32.

33. Mendis-Handagama SMLC, Siril Ariyaratne HB. Leydig cells, thyroid hormones and steriodogenesis. Indian $J$ Exp Biol 2005:43:939-62.

34. Kaphale K, Wu LS, Tsai YF. Effects of putative neurotransmitters on testosterone production from in vitro mice interstitial cells culture. J Anim Vet Adava 2003;2:119-25.

35. Fait G, Vered Y, Yogev L, Gamzu R, Lessing JB, Paz G, et al. High levels of catecholamines in human semen: a preliminary study. Andrologia 2001;33:347-50.

Received April 3, 2007; accepted October 28, 2007 


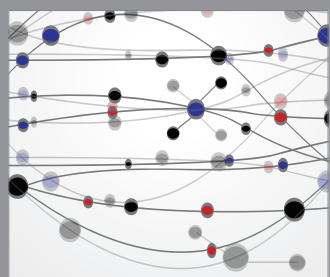

The Scientific World Journal
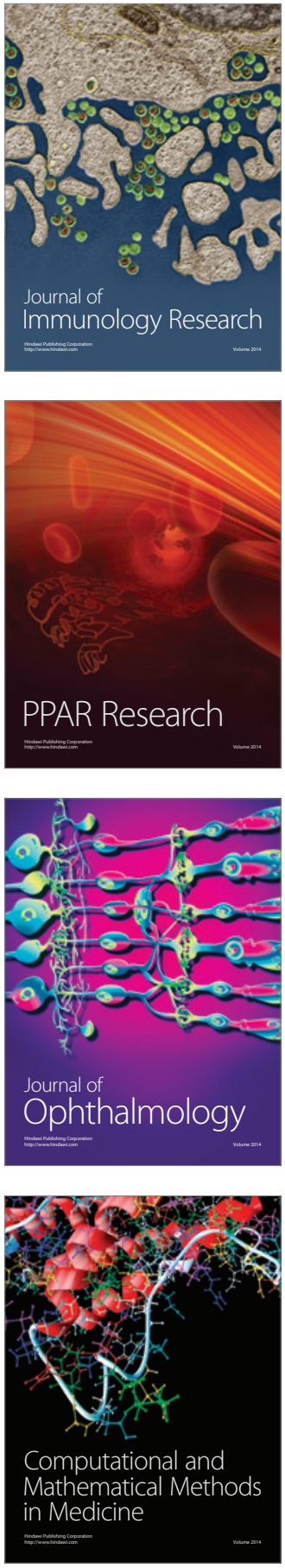

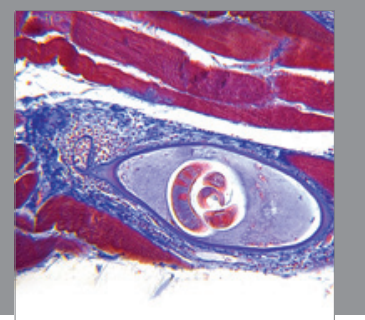

Gastroenterology

Research and Practice
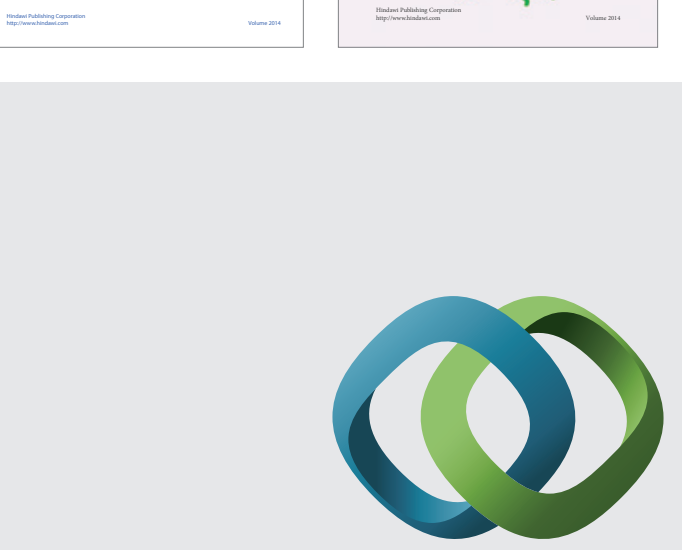

\section{Hindawi}

Submit your manuscripts at

http://www.hindawi.com
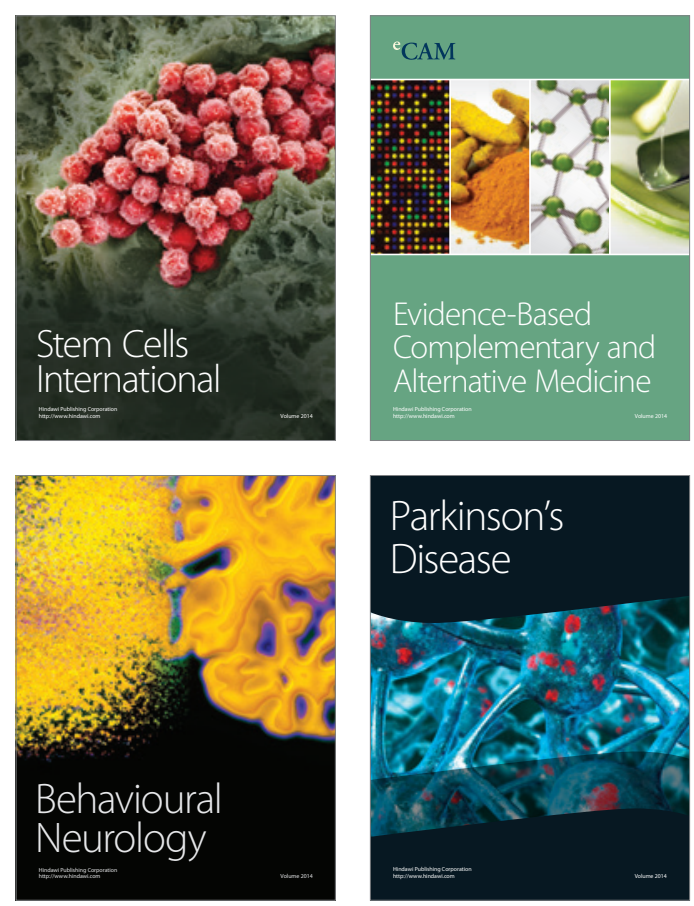

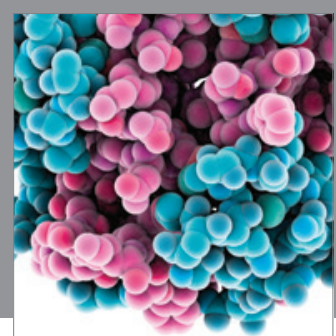

Journal of
Diabetes Research

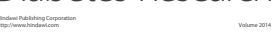

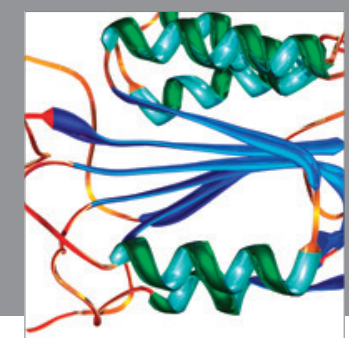

Disease Markers
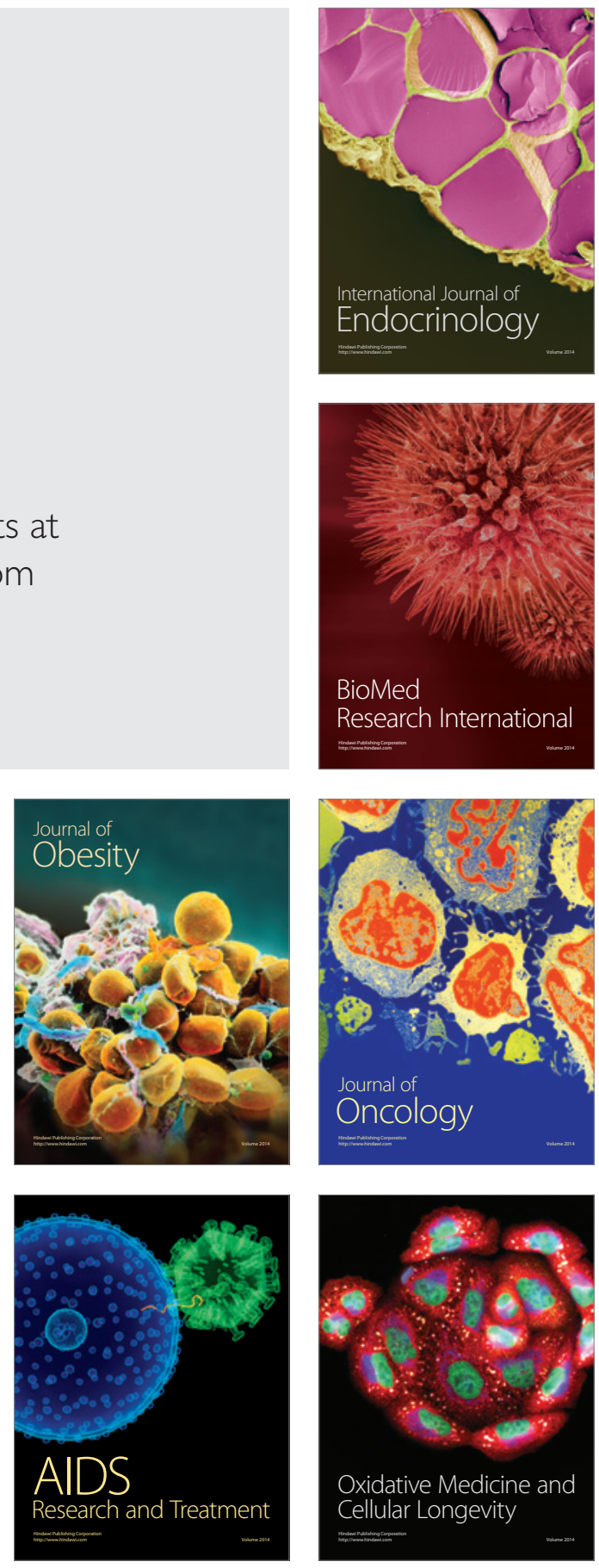